\title{
The Benefit of Split Nonlinearity Compensation for Single-Channel Optical Fiber Communications
}

\author{
Domaniç Lavery, Member, IEEE, David Ives, Gabriele Liga, Student Member, IEEE, \\ Alex Alvarado, Senior Member, IEEE, Seb J. Savory, Senior Member, IEEE, and Polina Bayvel, Fellow, IEEE
}

\begin{abstract}
In this letter, we analyze the benefit of digital compensation of fiber nonlinearity, where the digital signal processing is divided between the transmitter and the receiver. The application of the Gaussian noise model indicates that, where there are two or more spans, it is always beneficial to split the nonlinearity compensation. The theory is verified via numerical simulations, investigating the transmission of a single-channel 50-GBd polarization division multiplexed 4- and 256-ary quadrature amplitude modulation signals over $100-\mathrm{km}$ standard singlemode fiber spans, using lumped amplification. It is shown, theoretically, that the signal-to-noise ratio gain for long distances and high bandwidth transmission is $1.5 \mathrm{~dB}$ versus transmitteror receiver-based nonlinearity compensation.
\end{abstract}

Index Terms-Coherent optical communications, quadrature amplitude modulation (QAM), nonlinearity compensation.

\section{INTRODUCTION}

$\mathbf{R}$ ECENT efforts in overcoming the limit to optical communications imposed by fiber nonlinearity can be broadly grouped into two areas: optical and digital techniques. Optical techniques include, for example, optical phase conjugation (OPC) using twin waves [1] or OPC devices placed mid-span [2]. Digital techniques include transmitter- [3], [4] and receiver-side [5] digital nonlinearity compensation (NLC), simple nonlinear phase shifts [6], [7], perturbation-based precompensation [8], adaptive filtering [9] and optimum detection [10]. With the exception of optimum detection (a special case of receiver-side NLC for single span transmission) the digital signal processing (DSP) techniques are algorithms which invert the propagation equations for the optical fiber, either exactly or with simplifying approximations.

Consider the model in Fig. 1, which shows a transmission link with digital NLC at both the transmitter and the receiver. To date, the best performing experimentally demonstrated digital technique for receiver-side digital NLC is the digital backpropagation (DBP) algorithm. This algorithm numerically

Manuscript received April 6, 2016; revised May 3, 2016; accepted May 19, 2016. Date of publication May 24, 2016; date of current version June 29, 2016. This work was supported by the U.K. Engineering and Physical Sciences Research Council (EPSRC) under Grant EP/J017582/1 (UNLOC) and Grant EP/L026155/1 (INSIGHT).

D. Lavery, G. Liga, A. Alvarado, and P. Bayvel are with the Optical Networks Group, Department of Electronic and Electrical Engineering, University College London, London WC1E 7JE, U.K. (e-mail: d.lavery@ee.ucl.ac.uk; gabriele.liga.11@ucl.ac.uk; alex.alvarado@ieee.org; p.bayvel@ucl.ac.uk).

D. Ives and S. J. Savory were with the Optical Networks Group, Department of Electronic and Electrical Engineering, University College London, London WC1E 7JE, U.K. They are now with the Electrical Engineering Division, Department of Engineering, University of Cambridge, Cambridge CB3 OFA, U.K. (e-mail: di231@cam.ac.uk; sjs1001@cam.ac.uk).

Color versions of one or more of the figures in this letter are available online at http://ieeexplore.iee.org.

Digital Object Identifier 10.1109/LPT.2016.2572359

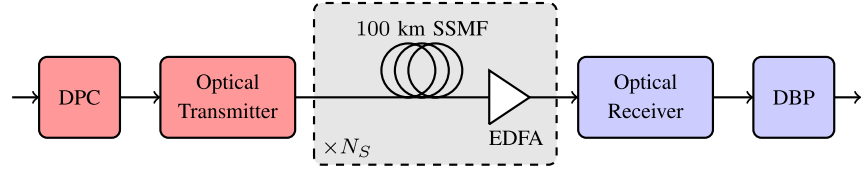

Fig. 1. Transmission model used for investigating the performance of fiber nonlinearity compensation, where the digital nonlinearity compensation is divided between transmitter and receiver.

solves the inverse of the optical fiber propagation equations to compensate the linear and nonlinear impairments introduced by the optical fiber transmission; albeit not taking account of amplifier noise. ${ }^{1}$ The demonstrations of multi-channel NLC via receiver-side DBP [5] which takes account of the interchannel nonlinear distortions, have recently been repeated using similar digital techniques to predistort for nonlinearity at the transmitter - digital precompensation (DPC) [3]. We note that, as might be expected due to the symmetry of the transmission link, ${ }^{2}$ the experimentally demonstrated performance of the pre- and post-compensation algorithms is similar; achieving a $100 \%$ increase in transmission reach [3], [5].

Whether applying DBP or DPC, additive noise from in-line optical amplifiers is enhanced, which limits the performance of the NLC. One can, therefore, make an argument for dividing the NLC equally between transmitter and receiver, in that it limits the noise enhancement in the compensated waveform to the signal-noise interaction present at the center (rather than the end) of the transmission link.

Although split NLC (dividing digital NLC between transmitter and receiver) has previously been considered, experimental implementation was confined to the special case of simplified DSP (single nonlinear phase shift [6]) and theoretical analysis considered only residual NLC after OPC [2]. In this Letter, we assess the performance of split NLC via numerical simulations, and characterize performance in terms of achievable signal-to-noise ratio (SNR). Further, we confirm these results theoretically.

\section{Model of Transmission Performance Using DIGITAL NONLINEARITY COMPENSATION}

To model the effect of digital NLC we used a coherent Gaussian noise (GN) model of nonlinear interference [12]

\footnotetext{
${ }^{1}$ DBP is a zero-forcing equalization, however it should be noted that algorithms such as stochastic DBP incorporate the noise distribution to improve the NLC performance [11].

${ }^{2}$ As noted in [7], the first span is an exception to link symmetry in that, under the simplifying assumptions of a noiseless transmitter, no polarization mode dispersion and no photon-phonon interactions, the nonlinear interference in this span can be compensated exactly with DPC.
} 
TABLE I

Summary of System Parameters USEd IN Fiber SimUlation

\begin{tabular}{l|r|l}
\hline Parameter & Value & Units \\
\hline Fiber attenuation & 0.2 & $\mathrm{~dB} / \mathrm{km}$ \\
Dispersion parameter & 17 & $\mathrm{ps} /(\mathrm{nm} \cdot \mathrm{km})$ \\
Fiber nonlinear coefficient & 1.2 & $1 /(\mathrm{W} \cdot \mathrm{km})$ \\
Span length & 100 & $\mathrm{~km}$ \\
Simulation step size & 100 & $\mathrm{~m}$ \\
NLC step size & 100 & $\mathrm{~m}$ \\
Symbol rate & 50 & $\mathrm{GBd}$ \\
EDFA noise figure & 5 & $\mathrm{~dB}$ \\
Pulse shape & RRC, $1 \%$ & rolloff \\
\hline
\end{tabular}

including the effect of signal-ASE (amplified spontaneous emission) noise interactions. The model treats the field propagating in the fiber as a summation of signal and noise fields, incorporating the signal-ASE noise interaction as a form of cross channel interference. Similar to [13] the SNR at the receiver is approximated as

$$
\mathrm{SNR} \approx \frac{P}{N_{S} P_{A S E}+N_{S}^{1+\varepsilon_{s s}} \eta_{s S} P^{3}+3 \xi \eta_{s n} P^{2} P_{A S E}},
$$

where $P$ is the signal power, $N_{S}$ is the number of spans, $P_{A S E}$ is the ASE noise power in the signal bandwidth from a single span amplifier, $\eta_{s s}$ is a single span nonlinear interference factor for the self channel interference (i.e., signalsignal interactions), $\eta_{s n}$ is a single span nonlinear interference factor for signal-squared ASE noise interference, $\varepsilon_{s s}$ is the coherence factor for self channel interference and $\xi$ is a factor depending on the number of spans and the method used for digital NLC. The nonlinear interference terms with ASE noise squared and cubed have been neglected as insignificant. In order to analytically calculate the SNR when applying different nonlinear equalization methods, the $\xi$ parameter must be computed for DPC, DBP and split NLC $\left(\xi_{D P C}, \xi_{D B P}\right.$ and $\xi_{S C}$, respectively).

Following the method in the Appendix, it is found that the difference in SNR at optimum signal launch power when applying split NLC versus DBP is given by

$$
\Delta \mathrm{SNR}=\sqrt{\frac{\xi_{D B P}}{\xi_{S C}}},
$$

where $\xi_{D B P}$ and $\xi_{S C}$ are given in the appendix by Eqs. (6) and (7). Choosing a 50\% transmitter:receiver split ratio for NLC, and for a large number of spans, (2) becomes

$$
\lim _{N_{S} \rightarrow \infty} \Delta \mathrm{SNR}=\sqrt{2^{1+\varepsilon_{s n}}} .
$$

For this work, $\varepsilon_{s n}=0.134$, obtained via numerical integration (see appendix). For $\varepsilon_{s n}$ varying from 0 to 0.3 (a conservatively high value) $\triangle \mathrm{SNR}$ varies between $1.5 \mathrm{~dB}$ and $1.95 \mathrm{~dB}$. In the large bandwidth limit, $\varepsilon_{s n}$ tends to zero.

\section{Numerical Simulations}

Consider the point-to-point transmission link shown in Fig. 1, consisting of an idealized optical transmitter and coherent receiver separated by $N_{S}$ spans of standard single mode fiber (SSMF), followed by erbium doped fiber amplifiers (EDFA). The simulation parameters are shown in Table I.
The transmitted signal was single channel $50 \mathrm{GBd}$ polarization division multiplexed (PDM) 4- and 256-ary quadrature amplitude modulation (QAM). These formats were chosen to investigate the validity of the model when using NLC for both low and high cardinality modulation. The signal was sampled at 4 samples/symbol (to take account of the signal broadening due to fiber nonlinearity) and shaped using a root-raised cosine (RRC) filter. Where DPC was considered, it was applied at this point using the split step Fourier method (SSFM) to solve the Manakov equation [14, eq. (12)].

The optical fiber span was again modeled by solving the Manakov equation using the SSFM. Each fiber span was followed by an EDFA which applied gain which exactly compensated the previous span loss.

Where required, the receiver DSP applied either frequency domain chromatic dispersion compensation (linear case), or DBP. Subsequently, a matched RRC filter was applied to the signal, and the signal was downsampled from 4 to 1 sample/symbol. To mitigate any residual phase rotation due to uncompensated nonlinear interference, carrier phase recovery was performed as described in [15]. Finally, the SNR was estimated over $2^{17}$ symbols by comparing the transmitted and received symbols, using the SNR definition in [15].

\section{RESULTS AND DisCUSSION}

The analytical expression for SNR, (1), was evaluated using the methods outlined in the Appendix for calculating $\xi$ for both linear signal equalization (CDC) and for each NLC technique: DPC, DBP and split NLC. In simulation, transmission distances were considered between 200 and $10000 \mathrm{~km}$ (2-100 spans), with the signal launch power varied in $1 \mathrm{~dB}$ steps. For each transmission distance, the SNR was determined at the optimum launch power. Fig. 2(a) shows how the maximum achievable SNR for a PDM-4QAM signal varies with transmission distance when applying different digital NLC techniques. A 50\% split ratio is used for split NLC. It should be noted that there is excellent agreement between the analytical expressions and the SSFM simulations, with an SNR estimation agreement better than $0.4 \mathrm{~dB}$ for all distances when NLC is applied. For linear CDC, the model accuracy increases for distances over approximately $1000 \mathrm{~km}$ where the GN model is known to have greater accuracy due to the high accumulated chromatic dispersion. An SNR improvement for split NLC over both DBP and DPC at all distances is also observed, as predicted by (3).

Fig. 2(b) shows the PDM-256QAM transmission performance. The increased cardinality modulation improves the accuracy of linear CDC model at short distances, as expected. Note that this has no impact on the high accuracy of the NLC performance model, which can be attributed to the compensation of signal-dependent self-interference terms.

The results in Fig. 2(c) show the SNR gain that can be achieved by dividing the NLC between transmitter and receiver with different ratios. Note that the gain of DPC over DBP rapidly diminishes with transmission distance. Further, it can be seen that a 50\% NLC split ratio is optimum for all transmission distances. 

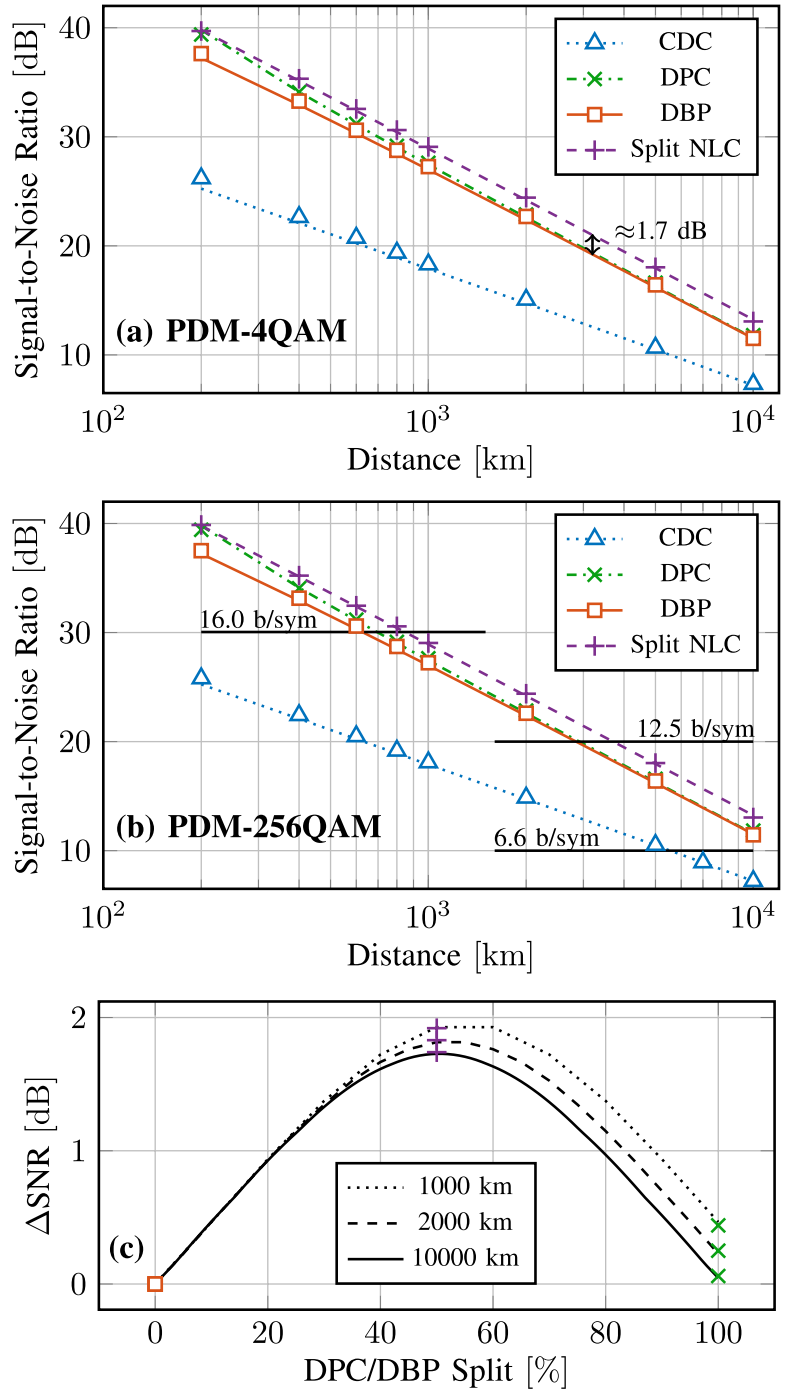

Fig. 2. Analytical (curves) and simulated (markers) transmission performance when applying different fiber nonlinearity compensation methods. SNR (over signal bandwidth) vs. transmission distance is shown for (a) PDM-4QAM and (b) PDM-256QAM. Horizontal lines indicate the mutual information of the PDM-256QAM signal at each decade of SNR. The mutual information is calculated from SNR under a Gaussian noise assumption, as in [15]. (c) SNR gain over DBP for PDM-256QAM when varying NLC split ratio.

It should be noted that the SSFM simulations and the theoretical analysis represent a somewhat idealized model of an optical fiber transmission system. For example, polarization mode dispersion is known to negatively impact on the performance of digital NLC, and yet is not considered in this model. Therefore, these results should be interpreted as an optimistic estimation of performance using digital NLC. Nevertheless, this work demonstrates that the current arrangements of digital NLC (DPC or DBP) can be substantially improved.

\section{Conclusions}

We used a closed form approximation for the accumulation of the signal-ASE interaction over multiple spans in order to analyse the potential SNR gain when dividing NLC between transmitter and receiver. The optimum launch power, and hence SNR gain, when using split NLC will increase by $1.5 \mathrm{~dB}$ with respect to both DPC and DBP in the limit of long distance, high bandwidth transmission. Split NLC is shown, both theoretically and by numerical simulation, to globally outperform both DPC and DBP for all transmission distances. Although this Letter covers theoretical aspects of split NLC, the SNR gains warrant further investigation into implementation issues, such as computational complexity.

\section{APPENDIX}

The following is a derivation of analytical expressions for $\xi$ in the case of both linear $\mathrm{CDC}$ at the receiver, and nonlinear compensation using pre-, post- or split-NLC. The nonlinear interference factors, $\eta_{s s}$ and $\eta_{s n}$, were calculated using numerical integration of the $\mathrm{GN}$ model reference equation [12, eq. (1)]. Note $\eta_{s n} \approx \eta_{s s}$ but is more accurately given by numerical integration of $[16$, eq. (7)] where the spectral shape, $g\left(\dot{f}_{1}+\dot{f}_{2}+f\right)$, is replaced by unity to represent the uniformity of the ASE spectrum. Coherence factors $\varepsilon_{s s}$ and $\varepsilon_{s n}$ were calculated by obtaining the nonlinear interference factors for 100 spans by numerical integration and using

$$
\varepsilon=\frac{\log \left(\frac{\eta_{100}}{\eta_{1}}\right)}{\log (100)}-1
$$

where $\eta_{1}$ is the single span nonlinear interference factor and $\eta_{100}$ is the nonlinear interference factor for 100 spans. In each case, $\eta$ is substituted by $\eta_{s s}$ or $\eta_{s n}$, as appropriate. Note that the purpose of $\varepsilon$ is to change the coherence of the interference terms, altering the accumulation of the nonlinear interference with number of spans, and that $0 \leq \varepsilon \leq 1$.

The effect of NLC on the effective received SNR is modeled by assuming that NLC effectively subtracts in power the nonlinear interference generated by the forward propagating field. This simplification is customary in the literature and can be seen as the result of two assumptions: i) a perturbative firstorder approximation, and ii) uncorrelation of all the optical fields involved in the SNR calculation. Note that this implicitly assumes that NLC is applied to the full received optical field. As shown in [17], DBP generates a first-order field, identical, but with opposite sign, to the forward-propagated field, provided that the linearly-propagated field (zeroth-order solution) along the fiber link is the same, hence the cancellation. However, due to the noise accumulation over the link, there is a mismatch between the linearly forward-propagated field and the backward-propagated field (or the precompensated field). As a result, residual signal-ASE interaction terms are still present after the application of either DBP or DPC, representing one of the main performance limitations [18].

Further assuming a weak nonlinear interaction between ASE noise contributions along the link, ${ }^{3}$ the calculation of the signal-ASE interaction terms can be performed by considering each ASE noise contribution as separately interacting with the signal in each span.

The nonlinear interference scaling coefficient, $\xi$ accounts for the noise generated due to this signal-ASE interaction. In the case of linear CDC, each contribution of ASE noise

\footnotetext{
${ }^{3}$ Indeed, the perturbative approximation does not allow the additivity of nonlinear terms arising from two or more optical fields adding together, even to the first order.
} 
interacts with the signal from the span following its addition, up until the end of the link. In the configuration analysed herein (Fig. 1), the first ASE noise contribution interacts with the signal in the second span. Likewise, in the case of DPC, signal-ASE noise interference accumulates from the second span onwards, since the first noise source follows the first span. Thus, for the CDC and DPC scenarios,

$$
\xi_{D P C}=\xi_{C D C}=\sum_{k=1}^{N_{S}-1} k^{1+\varepsilon_{s n}} .
$$

For DBP, noise from the last amplifier will be backpropagated as if it were signal for $N_{S}$ spans. The noise from the penultimate amplifier will have interacted with the signal for one span but will be backpropagated as if it were signal over all $N_{S}$ spans. Thus the signal noise interaction over the final span will be correctly compensated, leading to $N_{S}-1$ spans of excess nonlinear interference. Thus the total signal-ASE noise interference is given by the following sum over all spans

$$
\xi_{D B P}=\sum_{k=1}^{N_{S}} k^{1+\varepsilon_{s n}} .
$$

If the NLC is split between $N_{S 1}$ spans of DPC and $N_{S 2}$ spans of DBP such that the total number of spans is $N_{S}=$ $N_{S 1}+N_{S 2}$, then $\xi_{S C}$ is given by a combination of (5) and (6) as

$$
\xi_{S C}=\sum_{k=1}^{N_{S 1}-1} k^{1+\varepsilon_{s n}}+\sum_{k=1}^{N_{S 2}} k^{1+\varepsilon_{s n}} .
$$

The advantage of splitting the compensation arises since $\xi_{D B P}$ and $\xi_{D P C}$ increase superlinearly with the number of spans. $\xi_{S C}$ is minimized for $N_{S 1}=\left\lceil\frac{N_{S}}{2}\right\rceil$ and $N_{S 2}=\left\lfloor\frac{N_{S}}{2}\right\rfloor$.

The SNR gain due to the split NLC can be quantified using an approximated closed-form expression for the summation, in each of (5) and (6). Using Faulhaber's formula [19, eq. (0.121)], such a summation can be expressed as

$$
\begin{aligned}
\sum_{k=1}^{N_{S}} k^{1+\varepsilon_{s n}}= & \frac{N_{S}^{2+\varepsilon_{s n}}}{2+\varepsilon_{s n}}+\frac{N_{S}^{1+\varepsilon_{s n}}}{2}+\frac{1}{2}\left(\begin{array}{c}
1+\varepsilon_{s n} \\
1
\end{array}\right) B_{2} N_{S}^{\varepsilon_{s n}} \\
& +\frac{1}{4}\left(\begin{array}{c}
1+\varepsilon_{s n} \\
3
\end{array}\right) B_{4} N_{S}^{\varepsilon_{s n}-2}+\ldots
\end{aligned}
$$

where the coefficients $B_{n}$ are known as the Bernoulli numbers. A sufficiently accurate closed-form for $N_{S}>1$ can be derived by truncating (8) to the first 2 terms. These terms rapidly dominate the higher order terms as $N_{S}$ increases, particularly considering that $B_{2}=1 / 6$ and $B_{4}=-1 / 30$. The SNR gain, $\triangle \mathrm{SNR}$, for split compensation over DBP can be defined as the ratio between the SNRs achieved by each compensation technique at optimum launch power. All NLC techniques remove the cubic terms in (1). Thus, considering that maximizing the SNR leads to the optimum launch power given by

$$
P_{o p t}=\frac{1}{2 P_{A S E} \sqrt{N_{S} \eta_{s n} \xi}}
$$

and that $\mathrm{SNR} \propto P$ at the optimum power since the overall ASE noise power is equal to the signal-ASE interaction power, the change in SNR is given by (2). Substituting the first two terms from the approximation (8) into (2), and choosing $N_{S 1}=\left\lceil\frac{N_{S}}{2}\right\rceil$ when calculating $\xi_{S C}$, for an asymptotically large number of spans, we obtain (3).

\section{ACKNOWLEDGMENT}

The authors thank Prof. A. Ellis and Dr. L. Galdino for discussions and comments on earlier drafts of this Letter.

\section{REFERENCES}

[1] X. Liu, A. R. Chraplyvy, P. J. Winzer, R. W. Tkach, and S. Chandrasekhar, "Phase-conjugated twin waves for communication beyond the Kerr nonlinearity limit," Nature Photon., vol. 7, no. 7, pp. 560-568, Apr. 2013.

[2] A. D. Ellis, M. E. McCarthy, M. A. Z. Al-Khateeb, and S. Sygletos, "Capacity limits of systems employing multiple optical phase conjugators," Opt. Exp., vol. 23, no. 16, pp. 20381-20393, Aug. 2015.

[3] E. Temprana et al., "Two-fold transmission reach enhancement enabled by transmitter-side digital backpropagation and optical frequency comb-derived information carriers," Opt. Exp., vol. 23, no. 16, pp. 20774-20783, Aug. 2015.

[4] K. Roberts, C. Li, L. Strawczynski, and M. O'Sullivan, I. Hardcastle, "Electronic precompensation of optical nonlinearity," IEEE Photon. Technol. Lett., vol. 18, no. 2, pp. 403-405, Jan. 15, 2006.

[5] R. Maher et al., "Reach enhancement of 100\% for a DP-64QAM superchannel using MC-DBP," in Proc. OFC Conf., Mar. 2015, paper Th4D.5.

[6] A. J. Lowery, "Fiber nonlinearity pre- and post-compensation for long-haul optical links using OFDM," Opt. Exp., vol. 15, no. 20, pp. 12965-12970, Oct. 2007.

[7] D. Lavery, M. Paskov, R. Maher, B. Thomsen, S. Savory, and P. Bayvel, "Low complexity multichannel nonlinear predistortion for passive optical networks," in Proc. Signal Process. Photon. Commun. Conf., May 2015, paper SpS2C.5.

[8] Y. Gao et al., "Reducing the complexity of perturbation based nonlinearity pre-compensation using symmetric EDC and pulse shaping," Opt. Exp., vol. 22, no. 2, pp. 1209-1219, Jan. 2014.

[9] M. Secondini and E. Forestieri, "On XPM mitigation in WDM fiber-optic systems," IEEE Photon. Technol. Lett., vol. 26, no. 22, pp. 2252-2255, Nov. 15, 2014.

[10] G. Liga, A. Alvarado, E. Agrell, M. Secondini, R. I. Killey, and P. Bayvel, "Optimum detection in presence of nonlinear distortions with memory," in Proc. ECOC, Sep. 2015, pp. 1-3, paper P.4.13.

[11] N. V. Irukulapati, H. Wymeersch, P. Johannisson, and E. Agrell, "Stochastic digital backpropagation," in Proc. IEEE Trans. Commun., vol. 62 , no. 11, pp. 3956-3968, Nov. 2014.

[12] P. Poggiolini, "The GN model of non-linear propagation in uncompensated coherent optical systems," J. Lightw. Technol., vol. 30, no. 24 pp. 3857-3879, Dec. 15, 2012.

[13] A. D. Ellis et al., "The impact of phase conjugation on the nonlinearShannon limit: The difference between optical and electrical phase conjugation," in Proc. IEEE Summer Topical Meeting, Nassau, Bahamas, vol. 2. Jul. 2015, pp. 209-210.

[14] D. Marcuse, C. R. Manyuk, and P. K. A. Wai, "Application of the Manakov-PMD equation to studies of signal propagation in optical fibers with randomly varying birefringence," J. Lightw. Technol., vol. 15, no. 9, pp. 1735-1746, Sep. 1997.

[15] A. Alvarado, E. Agrell, D. Lavery, R. Maher, and P. Bayvel, "Replacing the soft-decision FEC limit paradigm in the design of optical communication systems," J. Lightw. Technol., vol. 33, no. 20, pp. 4338-4352, Oct. 15, 2015

[16] D. J. Ives, P. Bayvel, and S. J. Savory, "Adapting transmitter power and modulation format to improve optical network performance utilizing the Gaussian noise model of nonlinear impairments," J. Lightw. Technol., vol. 32, no. 21, pp. 3485-3494, Nov. 1, 2014.

[17] M. Secondini, E. Forestieri, and G. Prati, "Achievable information rate in nonlinear WDM fiber-optic systems with arbitrary modulation formats and dispersion maps," J. Lightw. Technol., vol. 31, no. 23, pp. 3839-3852, Dec. 1, 2013.

[18] G. Gao, X. Chen, and W. Shieh, "Influence of PMD on fiber nonlinearity compensation using digital back propagation," Opt. Exp., vol. 20, no. 13, pp. 14406-14418, Jun. 2012.

[19] I. S. Gradshteyn and I. M. Ryzhik, Table of Integrals, Series, and Products, 6th ed. San Diego, CA, USA: Academic, 2000 